\title{
Osnabrück au pied de la lettre
}

En littérature, que serait-ce que prendre les mots « au pied de la lettre », expression paradoxalement métaphorique pour évoquer un emploi littéral du langage ? Dans la mesure où l'accent mis sur le sens strict des mots a pour effet de privilégier le régime de la signification, l'on peut dire qu'une telle approche va à l'encontre de la poésie. Néanmoins, dans la mesure où il s'agit de prendre les mots justement à la lettre, littéralement, l'importance donnée au corps même du mot présente des similitudes avec la sensibilité à la forme et aux considérations formelles qui caractérise le régime littéraire. Si donc une démarche littérale s'écarte du poétique en donnant la priorité au sens propre sur le figuré, elle s'en rapproche en privilégiant le concret sur l'abstrait. J'inscris au départ cette question du rapport entre littéralité et littérarité parce qu'elle me semble offrir une perspective fructueuse pour aborder Osnabrück ${ }^{1}$, livre dominé par le souci de dresser un portrait fidèle d'Ève, la mère de la narratrice. Ève, nous allons le voir, est la reine de la locomotion, aussi bien à pied qu'en empruntant divers moyens de transport. Dans le contexte de ce livre, le fait que les étymologies latine et grecque des mots «transport» et «métaphore » se traduisent littéralement $($ méta $=$ trans $;$ pherein $=$ portare $)$ donne à penser. Quel rapport Cixous proposet-elle entre le déplacement physique et le déplacement du sens ? Quel rapport faut-il lire entre les voyages de la mère et le transport qui importe le plus pour la narratrice, le «transport de l'âme » de l'expérience de la «magie spirituelle de l'art » (p. 79) ?

L'une des grandes différences entre la narratrice d'Osnabrück et sa mère concerne justement la valeur à attribuer à différentes pratiques du langage. L'utilité est en général le critère de choix d'Ève. Bien qu'elle ne croie pas en Dieu, elle juge que « Dieu est une chose assez utile », vu que l'homme a besoin de «s'adresser à quelque chose » (p. 33). Quelque défi que la vie lui réserve - mort du père quand elle était encore enfant, mort du mari la laissant seule avec deux jeunes enfants - elle ne retourne jamais en arrière, estimant : «Le chagrin est inutile. On a déjà eu le souci » (p. 33). Elle a formé son corps à son idée, « exact, utile, modéré, non charnel» (p. 35). Aussi n'est-il pas surprenant que cette valorisation de l'utile

\footnotetext{
${ }^{1}$ Hélène Cixous, Osnabrück, Paris, Éditions des femmes, 1999.
} 
influe sur son rapport à la langue. Par la juxtaposition de leurs deux paroles, Cixous souligne le contraste entre le discours exubérant, excessif de la narratrice et le discours simple de sa mère :

\footnotetext{
Je descends de ma mère ou bien au contraire je suis son élève, l'élève d'Ève, élevée que je suis, emportée par une élévation contraire.

ÈVE : Je suis terre à terre. (p. 43-44)
}

Plus encore que le contraste sémantique entre le haut et le bas (ou plus précisément entre, d'une part, l'incertitude du haut et du bas créée par l'emmêlement de la descente et de l'élévation et, d'autre part, le bas terrestre), c'est le jeu du signifiant - notamment la visibilité exorbitante des lettres du nom d’Ève dans «élève », «élevée », «élévation » - qui accuse l'écart entre la parole de la narratrice et celle de sa mère. Ève se dit terre à terre d'une manière elle-même terre à terre : c'est-à-dire, selon la définition du Petit Robert, "sans s'éléver du niveau commun », «peu poétique $»^{2}$.

Cela nous invite à lire dans l'épigraphe que Cixous place au seuil de son texte, épigraphe peu habituelle parce que prosaïque au plus haut degré, une façon d'inscrire Osnabrück sous le signe de sa mère. Le livre, dont le premier chapitre commence par une méditation sur l'endroit où il devrait commencer, commence en fait par un extrait long d'une page et demie de l'entrée sur Osnabrück du Grand Dictionnaire universel de Larousse. Au niveau du sens, en renseignant le lecteur sur le lieu d'origine de la mère (coordonnées géographiques de la ville, industries principales, etc.), l'épigraphe fournit une leçon concise de la géographie qu'Ève dira préférer à l'histoire (p. 102). Mais l'épigraphe est précurseur également dans la mesure où l'encyclopédie offre l'exemple suprême d'un discours factuel, informatif, où le langage fonctionne comme un outil de communication. La mise en exergue de l'extrait rend hommage à la mère non seulement donc au niveau des associations thématiques mais en faisant valoir un langage « terre à terre ».

À cet égard, l'encyclopédie se situe aux antipodes (du latin antipodus, « dont les pieds sont situés à l'opposé ») de la littérature. Vu la valeur attribuée par la mère à l'utilité, il n'est guère étonnant qu'elle ait un rapport très différent de sa fille à la littérature : «INUTILITE DU TEXTE POUR ELLE » (p. 43). Ève n'est tentée ni par le rêve ni par l'imagination d'un nouveau monde spirituel. Selon la narratrice, «l'âme est une maladie qu'elle n'a pas, [...] et qu'elle réprouve » (p. 22); ce qui l'intéresse, c'est la réalité. Pour elle, les choses sont ce qu'elles sont, ni plus ni moins, et elle aussi :

\footnotetext{
${ }^{2}$ Paul Robert, Le Petit Robert 1, Paris, Robert, 1992, p. 1948.
} 
Je suis contemporaine de la réalité, dit ma mère. Je suis moi, dit Ève. [...]

Une journée de ma mère compte vingt-quatre heures, pensé-je, la force de ma mère fait que les jours de ma mère mesurent vingt-quatre heures. Mes jours sont de durée imprévisible et inégale. (p. 33-34)

Le goût maternel de l'exactitude des choses et de la netteté des limites contraste ainsi fortement avec l'impulsion qui porte la narratrice vers l'ébranlement de toute identité, contraste qui se prolonge dans leur rapport au texte littéraire. Alors que la narratrice se sent fondamentalement appelée par ce qui remet en cause son identité plutôt que par ce qui la consolide, se laissant notamment conduire par «cette part-Ève de moi qui me reste inconnue » (p. 99), la mère préfère rester « maître de son bord »,

de crainte si elle se laissait aller à l'intérieur, de perdre le poste de commandante. C'est ainsi qu'elle lit pastout Zola ou pastout Proust ou ce que je lui ouvre à lire en fermant d'abord soigneusement le livre et la porte, puis ayant chaussé ses patins à glace, elle glisse à toute vitesse par-dessus le texte sans jamais tomber sur les genoux. Elle saute les descriptions les passages descendants les montées les haltes les couloirs les coupe-gorge, tout l'inutile et elle va droit à l'essentiel. Tant qu'elle a travaillé elle n'a pas lu. [...] On n'avait pas le temps. C'est plutôt Somerset Maugham. Ce qui l'intéresse dans un livre c'est l'aventure, Conrad. La psychologie. (p. 100)

La préférence de la mère pour l'aventure et pour la psychologie est l'index d'une conception instrumentaliste du langage; il semble que la littérature fonctionne pour elle comme un outil de communication dont la valeur consiste dans le pouvoir de représentation. En effet, il est question à plusieurs égards ici de pouvoir, de maîtrise. De même que le désir de rester en contrôle d'elle-même (ne pas «perdre le poste de commandante ») signife une hiérarchie psychique implicite de la fermeté sur la faiblesse, Ève hiérarchise le livre, donnant la priorité à l' « essentiel » sur l'« inutile ». Or, lire de cette manière n'a justement rien d'une démarche littérale. Ni d'une marche métaphorique. Ève n’y va pas à pied, elle prend des « patins » pour glisser «par-dessus le texte », évitant ainsi le contact intime qui lui permettrait de connaître (et d'être touchée par) les éléments textuels ne servant pas à avancer l'action. Cela tranche évidemment avec l'attention détaillée et caressante que la narratrice voue aux mots. Cependant, il faut noter que la phrase où celle-ci décrit le patinage maternel participe elle-même au glissement : «Elle saute les descriptions les passages descendants les montées les haltes les couloirs les coupe-gorge ». Le manque de ponctuation essoufflé reproduit la sensation d'une course énergique qui nivelle les accidents du terrain en passant si rapidement que les distinctions locales s'embrouillent. En plus de signaler ce qui la différencie de sa mère, la narratrice saisit alors dans l'écriture l'occasion de lui emboîter le pas. 
Ce rapport double à la fois de distance et d'imitation en ce qui concerne Ève trouve son apogée lors de deux séquences élaborées qui empruntent des figures de déplacement pour mettre en regard la démarche de la narratrice avec celle de sa mère. De telles figures abondent dans Osnabrück. Les patins à glace font partie de toute une série de moyens de déplacement qui permettent à la mère de poursuivre son chemin vers «l'essentiel ». Son peu d'intérêt pour l'investigation spirituelle amène sa fille à peindre son espace psychique comme une aide au voyage à l'extérieur : «Sur le mur intérieur, une carte des réseaux de transports urbains. Ses amis les autobus et les métros » (p. 67). La mère est toujours en mouvement : «Elle va, Èva, Èvant» (p. 55), et tous les transports lui sont associés : le train, l'avion, le vélo, même l'ascenseur. Relativement tôt dans le livre, la narratrice décrit le rapport au transport comme site emblématique de la différence entre elle et sa mère :

Ève et moi nous courons pour prendre le train le même train, c'est alors qu'on voit comme tout nous sépare rien ne convient moi je retiens un taxi, elle veut le renvoyer, [...] elle n'aurait jamais fait ce que je fais, [...] nous arrivons à la gare où il est trop tôt, j'ai tort et elle a raison, à quoi bon lui dire, cela ne changerait rien, car dans le pays à mots elle ne vient pas, elle a horreur de prendre un taxi que j'ai commandé, elle a horreur des chauffeurs, dans la voiture elle me conduit, tourne le volant ou je descends freine, prends à gauche, ne double pas, c'est la dernière fois que je prends un transport avec toi (p. 46).

Train, taxi, voiture : même en prenant ensemble un transport en commun, la narratrice et sa mère sont séparées par le voyage qu'elles partagent. Toutefois, la netteté de la séparation est démentie par les deux longues séquences mentionnées-annoncées ci-dessus, qui invitent le lecteur à explorer Osnabrück (le premier livre que Cixous ait consacré à sa mère ${ }^{3}$ ) comme un voyage qui double les déplacements maternels autant qu'il s'y oppose.

La première séquence [j'ai l'impression que le sens du mot ici n'a pas très clair pour le lecteur: serait-il possible de spécifier à quoi il se réfère ? En effet, les deux séquences mentionnées juste avant sont à des pages différentes de celles décrites ensuite] commence quand la narratrice «craque » devant le désarroi de sa mère à ne pas pouvoir se rappeler le nom de sa tante et qu'elle accepte pour la première fois de transcrire les souvenirs d'enfance d'Ève. L'écriture introduit d'emblée le motif du transport : «Tout commence par le tramway d'Osnabrück » (p. 131). Au commencement, donc, est le mouvement, mouvement qui affecte jusqu'au « tout » qui commence déjà lui-même en déplacement, l'absence de guillemets dans

\footnotetext{
${ }^{3}$ En 1991, Cixous avait notoirement dit : «Il me semble que nous ne pouvons pas écrire de notre mère. J'en suis sûre. C'est une des limites de l'écriture » («En octobre 1991... », in Du Féminin, éd. par Mireille Calle-Gruber, Sainte-Foy et Grenoble, Le Griffon d'argile/Presses Universitaires de Grenoble, « Trait d'union »,1992, p. 134). Osnabrück représente le moment charnière à partir duquel la mère devient le personnage principal de ses écrits, contradiction à laquelle l'auteur elle-même réfléchit à de nombreuses reprises (voir par exemple Tours promises, Paris, Galilée, 2004, p. 95-96).
} 
le texte ne permettant pas au lecteur de distinguer avec certitude si c'est la mère qui prend ici la parole ou la fille qui continue son récit. Ensuite, au lieu de nous expliquer ce rôle inaugural du tramway, le texte dévie tout de suite pour décrire un autre réseau de communication, ou plutôt un début de réseau de communication, un réseau qui ne marche pas (encore). La grandmère d'Ève était seule dans Osnabrück à avoir le téléphone. Comme le souligne le deuxième paragraphe de la séquence dont l'italique nous invite à l'attribuer l'énonciation à la narratrice plutôt qu'à Ève, il était donc parfaitement inutile : «personne d'autre que mon arrière-grandmère n'ayant le téléphone à Osnabrück elle ne pouvait téléphoner qu'à Dieu en attendant » (p. 132). Cependant, «ce téléphone absolu est mon legs» (p. 131) : bien que, pratiquement parlant, ce téléphone ne rende possible aucun contact avec personne, il aurait permis à Helene Jonas non seulement de s'entretenir avec « Dieu » mais aussi d'établir un rapport à travers les générations avec son arrière-petite-fille Hélène. Étant donnée l'importance du motif du téléphone dans l'œuvre de Cixous ${ }^{4}$, il va sans dire que l'on peut voir dans cette image d'un «téléphone absolu», intransitif, branché sur une ligne directe avec Dieu, une figure de l'écriture cixousienne elle-même. Pratiquement parlant, le téléphone ne sert à rien. Mais il peut servir à parler autrement que pratiquement.

Ce qui est en cause au commencement de cette longue séquence, c'est alors un réseau de réseaux qui échouent à communiquer, ou qui communiquent autrement. Car le tramway à son tour s'avère être l'occasion d'un usage peu fonctionnel. Quand ils venaient à Osnabrück, Herbert et Kurt Löwenstein, cousins d'Ève qui habitaient un petit village sans tramway aux alentours de la ville, «prenaient un billet de tram et ne sortaient plus du tram, parce que l'aventure ne se terminait pas à un terminus, ils allaient sur un seul billet toute la journée d'un terminus à l'autre » (p. 132). L'«aventure » qui commence n'a absolument rien de téléologique. Or, c'est à ce moment précis du texte que s'inscrit la première d'une vingtaine de propositions incises qui scandent la séquence : «dit ma mère ». Ce tramway qui fait tout commencer pour les « cousins de Gemen » donne le branle également à la mère, qui se lance en parlant dans une aventure analogue aux déplacements qu'elle raconte. Une fois partie, Ève déjà «ne pouvait plus s'arrêter » (p. 132); Cixous décrit son récit comme une course où Ève se trouve emportée par sa propre vitesse :

\footnotetext{
${ }^{4}$ Derrida était le premier à le signaler dans «H.C. pour la vie, c'est à dire », in Hélène Cixous, croisées d'une œuvre (Actes du Colloque de Cerisy-la-Salle, juin 1998), dir. par Mireille Calle-Gruber, Paris, Galilée, «La philosophie en effet », 2000, p. 25-27). Voir aussi Geoffrey Bennington, « Teleanalysis », Paragraph, $\mathrm{n}^{\circ} 36: 2$, «Cixous, Derrida, Psychoanalysis», 2013, p. 270-285.
} 
Mais tante Hete, dit ma mère sans ralentir le pas, était à Essen, Essen an der Ruhr tout près de Mülheim, tante Selma était à Hambourg et tante Paula était à Gemen bei Borken, dit ma mère de plus en plus vite, toujours un peu pressée et essoufflée elle n'était pas très jolie elle voulait se mettre du rouge aux joues [...] elle avait aussi une amie dont la fille était kleptomane des gens très bien, même dans les petites villes il y a de tout et les colliers ou le rouge à lèvres qui disparaissaient on savait : c'est la kleptomane. Kleptomane encore un qu'il faut ranger avec Unikum, pensai-je. Elle avait aussi, dit ma mère de plus en plus vite, une autre amie c'était une belle femme Frau Hellman qui avait toujours des Kümmerer, alors j'ai dit : ça suffit on peut peut-être arrêter, dis-je, c'est impossible dit-elle, quand on a tous les hommes qui s'occupent d'elle et le mari ne dit rien (p. 135 ; nous soulignons).

La course gagne jusqu'à la narratrice elle-même: alors que les trois premières propositions incises (mises en relief ci-dessus en caractère gras) servent à distinguer les deux niveaux d'énonciation, la dernière a plutôt pour effet de les embrouiller. Tandis que l'italique parait mettre le début nettement du côté de la narratrice - «alors j'ai dit : ça suffit on peut peut-être arrêter »- la suite ne se laisse pas attribuer avec clarté. Le «je » en romain de « dis-je » a-t-il le même référent que le «je » en italique de «j'ai dit» ou le romain « elle» de «dit-elle »? Dans le deuxième cas, l'impossibilité dont parle la mère relèverait non de sa propre incapacité à s'arrêter de parler mais plutôt de son impatience face aux plaintes de Frau Hellman. L'impossibilité appartiendrait au même plan d'énonciation que la kleptomanie évoquée par Ève : il s'agirait d'une impossibilité remémorée. Mais l'incertitude ne s'arrête pas là. Car si la mère parle du passé plutôt que du présent en disant «c'est impossible », la logique nous invite rétrospectivement à lire la partie en italique aussi comme appartenant au récit du passé. Au lieu de la narratrice invitant sa mère à s'arrêter, ce serait la mère qui se rappelle avoir proposé à Frau Kellman de se taire.

Cet enchevêtrement des niveaux de l'énonciation s'accélère au fur et à mesure que la séquence avance, en tandem, avec la vitesse avec laquelle la mère parle. Au paragraphe suivant, la course fait place au vélo :

Ça suffit dis-je on peut arrêter là dis-je, mais elle ne pouvait plus s'arrêter elle pédalait sans arrêt parce qu'une fois partie elle ne pouvait plus s'arrêter, le vélo filait il avait pour ainsi dire filé tout seul au premier coup de pédale qu'elle avait donné alors qu'elle n'avait jamais touché un vélo de sa vie à treize ans, [...] le vélo avait obéi et s'était mis à tourner sur la place autour de l'église [...] car elle avait oublié de demander comment s'arrêter, Grete Bloch dis-je, c'est étrange c'est le nom de la petite copine de Kafka - je voulais demander à ma mère s'il y avait un rapport ou une coïncidence, elle filait, Grete Bloch criais-je attends criait-elle je ne peux pas m'arrêter, c'était une jeune fille d'une grande beauté qui courait plus vite qu'un lapin (p. 136).

Cette fois, la narratrice est clairement l'énonciatrice des mots «ça suffit ». Cependant, il est moins clair qu'elle en soit l'origine. Ils peuvent lui avoir été soufflés par la citation précédente, de même que la narratrice n'est pas simplement à l'origine de la métaphore du vélo qu'elle emprunte pour caractériser le discours de sa mère, et qu'elle filera sur plusieurs 
pages (jusqu'à : «Ma mère mit le pied par terre et le vélo s'arrêta » (p. 143)). La fin de la citation suggère que cette métaphore est puisée dans le souvenir raconté par la mère de sa première promenade à bicyclette, expérience forcément antérieure au récit qu'elle en fait à sa fille, et où elle avait également pris son élan sans pouvoir s'arrêter.

L'embrouillement des plans de l'énonciation rejoint ainsi l'une des préoccupations principales de la séquence : la place de l'auteur. « Tu crois être l'auteur ?» (p. 145), médite la narratrice après s'être rendu compte qu'elle a fait exactement ce qu'elle ne voulait pas faire en encourageant sa mère à lui raconter ses histoires concernant «un grand nombre de personnages secondaires alors que les personnages de Shakespeare ne la retiennent pas un instant ». Elle reconnaît avoir animé les morts sans le vouloir : «moi j’ai sifflé les défunts de ma mère alors qu'un seul mort m'intéresse et c'est le Mien» (p. 145) ${ }^{5}$. Cela lui fait l'impression d'assister à un banquet de convives morts qui ne lui laissent rien à manger et, à son horreur, lui imposent une «lutte pour la survie» (p. 146). Non seulement donc la narratrice se trouve n'être «qu'une sur cent» (p. 146) au lieu d'être privilégiée en tant que l'auteur, et entraînée dans une scène d'appétits goulus des plus ordinaires, scène qui lui déplaît profondément, mais même à l'intérieur de ces paramètres limités, elle n'est pas en position de contrôle :

La réalité se rit de moi qui croyais être l'autre. Mon illusion majeure vacille vers la fin assise à l'extrémité nord d'une longue table de destin funèbre. Festin, dit ma mère, tu as fait une faute. Ah oui cette faute c'est la mienne, j'en suis l'auteur. Je me levai et j'annonçai à la famille qu'en tout cas pour les fautes je prétends que l'auteur c'est moi. (p. 147)

Elle n'est pas «l'autre» qu'elle croyait être - mais la faute indique qu'elle n'en demeure pas moins étrangère à elle-même. C'est l'autre insoupçonné en elle qui parle à sa place en remplaçant une lettre par une autre. L'auteur, c'est l'autre autre, l'autre imprévu.

Est-il donc possible de savoir qui commande, qui conduit? Le déplacement de l'autorité de l'auteur fait écho à celui de l'autorité de la mère. Quand c'est au tour d'Omi, sa propre mère, dans le récit d'Ève, la narratrice réfléchit au fait qu'Ève appelait sa mère grand-mère (Omi étant l'équivalent allemand de Mamie) dès la naissance de sa fille :

je n'assistai pas à la passation des noms et des mères, toutefois, tandis que ma mère pédalait sans discontinuer, je ne pouvais m'empêcher de penser que par ma naissance Omi avait été promue grand-mère ou bien démue ayant cessé du matin au soir d'être Mutti pour être réléguée plus haut dans la hiérarchie maternelle. (p. 137)

${ }^{5}$ Dans Gare d'Osnabrück à Jérusalem (Paris, Galilée, «Lignes fictives », 2016), Cixous revient de son propre gré sur cette histoire familiale pour laquelle elle dit dans Osnabrück avoir une «sincère antipathie » (p. 144). 
Loin de situer nettement les différents échelons les uns par rapport aux autres, la « hiérarchie maternelle » ne sépare pas la promotion de la «démotion », le haut du bas. Les sens opposés se continuent ; la hiérarchie elle-même se prolonge «sans discontinuer » dans la mesure où l'ajout d'une génération remet les rôles en mouvement, faisant que la fille se renverse en mère ${ }^{6}$. En outre, à la page suivante la grand-mère regagne l'autorité qu'elle avait perdue en étant «intronisée » sous le nom Omi, redevenant jeune fille quand il est question d'un séjour qu'elle a passé «chez un rabbin dont la femme était en couches » (p. 138) pour apprendre la cuisine et s'occuper de la maison : «Alors ce rabbin comme tous les rabbins a essayé, mais Omi put her foot down et dit: Vous voyez ce lac ? Vous, s'il m'arrive quelque chose chez vous, vous me trouverez dans ce lac» (p. 138). L'expression anglaise signifie faire acte d'autorité ; Omi s'affirme telle une mère en refusant justement de prendre la place d'une mère.

Or, « to put your foot down » signifie aussi appuyer sur l'accélérateur. En freinant, elle accélère. Dans ce texte, il n'y a aucune position d'autorité qui ne soit minée d'avance par ce sur quoi elle s'exerce ; entamer une démarche, c'est déclencher un processus dont on perd aussitôt le contrôle et qui risque d'aggraver l'état même qu'il cherchait à améliorer. D'où, selon la narratrice, la singularité et la nécessité de l'art :

il n'y a que l'art qui reste toujours frais le reste est légume sec pensai-je, même un assassinat une fois sec ça n'intéresse pas, mais ma mère, lorsqu'elle avait appuyé pour la première fois sur la pédale, avait lancé une course contre l'oubli des noms propres et l'on sait depuis mon noncle Freud qu'on ne peut humainement pas arrêter cette course, car la souffrance qui accompagnerait l'écrasement du mouvement fouisseur vital s'apparente à la souffrance éprouvée lors d'une fausse couche. (p. 141)

En cherchant à déterrer les noms des morts afin de les sauvegarder, la mère se trouve paradoxalement emportée dans une course jusqu'à la mort, car l'interruption de l'élan couperait net au «mouvement vital », s'apparenterait à une «fausse couche », échec de la tentative de faire vivre. Il semble que seul l'art arrive à garder vif ce « mouvement vital ». La différence de l'art, ce n'est pas que le processus ne parvient pas à une fin ; c'est plutôt qu'après qu'il s'arrête, ce qu'il a produit/déterré reste «frais », continue à son tour de vivre, de bouger.

\footnotetext{
${ }^{6}$ Pour une analyse de la «danse des générations » entre mère et fille, je me permets de renvoyer à mon article «The Place of the Mother: Hélène Cixous's Osnabrück», Paragraph, n 27 : 1, 2004, p. 6-20.
} 
Le véritable enjeu pour la narratrice, c'est donc la différence entre la production du vivant et l'aridité des «pierres tombales » (p. 140) que la mère vise à construire pour tous les membres de sa famille qui ont disparu. Notons que le texte change encore de vitesse vers la fin de la séquence quand, après la course et le vélo, l'écriture nous transporte soudain en voiture :

\footnotetext{
À ce moment-là nous roulions en voiture dans la forêt immense des vents noirs soufflaient et il n'y avait personne sauf des milliers d'arbres qui pliaient, plusieurs d'entre eux s'écroulèrent à bout de forces, et je pleurai. C'est ma mère, pensai-je, une forêt de créatures, des semblables magnifiques et soudain condamnés. (p. 148)
}

Cette promenade en voiture introduit l'aboutissement de la séquence, le moment où la mère se rappelle le dernier nom à lui avoir échappé. En comparant Ève à des «milliers d'arbres », « une forêt de créatures » qui touchent à leur fin, le texte souligne l'imminence de l'immobilité : ces arbres fixes mais qui «pli[ent]»sous les vents sont des vivants capables d'un mouvement limité qui annonce déjà sa propre fin, l'écroulement qui mettra un terme jusqu'à cette mobilité réduite. Si la narratrice pleure, c'est parce que l'acharnement de la mère est « condamné » à s'éteindre ; c'est en pleine forêt et au comble de l'agitation, au moment où elle atteint son plus grand mouvement, que l'énergie qu'Ève dépense à énumérer les noms des morts lui semble le plus mortellement futile.

Soulignons le «nous » qui « roulions »: le pronom implique la narratrice au même titre que sa mère dans l'excursion. Comment faut-il lire cette promenade inattendue en voiture ? Littéralement, comme un voyage diégétique que pourtant rien d'autre dans l'histoire n'annonce? Ou faut-il y voir une surenchère des métaphores narratives de locomotion, l'accélération justement de la licence poétique de ne pas prendre les mots à la lettre ? C'est comme si la narratrice, tout en se joignant à sa mère pour mener le projet d'exhumation des noms jusqu'au bout, se devait en même temps de satisfaire à l'exigence éthique d'y mettre du sien, d'inventer quelque chose au lieu de répéter servilement, mortellement, une série de mots «tombaux ».

Ce qui ressort ainsi de la longue séquence consacrée à la comméroration maternelle des morts, c'est que l'art relève d'un déplacement vital. La métaphore fait bouger. Elle fait bouger jusqu'à la distinction entre métaphore et littéralité, d'après la deuxième séquence (p. 196-205) à exploiter le motif de la locomotion, séquence qui, elle, se déroule d'un bout à l'autre en voiture. À sa stupéfaction, la narratrice entend sa mère lui dire qu'elle va lui emprunter la voiture afin d'aller pour la première fois voir Omi au cimetière. Consciente du caractère sans 
précédent du projet («car au cimetière nous n’y sommes jamais allées » (p. 196)), la narratrice propose de l'accompagner : «Et un instant plus tard, sans aucune transition me voilà glissée sur le siège de ma voiture parmi mes nombreuses affaires sens dessus dessous et ma mère démarre » (p. 196). Cette fois l'équivalence du voyage et de la parole est explicite ; le récit de la mère «démarre » en même temps que la voiture. De plus, les vicissitudes du voyage et celles qu'Ève raconte au sujet de sa vie conjugale se font écho à plusieurs égards. Pendant la séquence, Ève fait état de diverses décisions prises par son mari (et ensuite par ses enfants) qui, selon elle, ont été catastrophiques. Elle reproche en particulier à son mari de ne pas être resté pédicure :

\begin{abstract}
S'il était resté pédicure, je n'aurais jamais été sage-femme, d'un autre côté, Tonfrère ne serait jamais devenu médecin, d'un autre côté tu n'aurais jamais écrit et passé ton temps dans le papier, tu me suis, dit ma mère, emportée par l'élan de ce qui aurait pu n'être pas et être, en avant, en arrière de chaque côté à une vitesse folle - quand on commence à entreprendre, on doit s'assurer devant soi et derrière, il est essentiel de savoir où l'on va mettre les pieds (p. 197).
\end{abstract}

Force est de constater que cette dernière expression se laisse prendre à la fois métaphoriquement et « au pied de la lettre ». D’abord, Ève en veut à son mari d'avoir voulu être médecin - «monsieur le Docteur» (p. 198) - sans en prévoir les conséquences, notamment les effets délétères du métier plus exigeant sur sa santé déjà fragile. Mais il avait négligé aussi de tenir compte de l'endroit où l'on allait littéralement «mettre les pieds », les problèmes rencontrés par la suite étant dus en partie au fait de s'être installé dans une «maison impossible» avec un «escalier sombre et très malodorant » qui a dissuadé les clients (p. 202). Elle lui reproche en somme d'avoir délaissé les avantages modestes des pieds (du bas) pour «la gloire» (le haut), alors que cela lui aurait été égal «d'être la femme d'un pédicure au lieu d'être la femme d'un médecin » (p. 198).

Tout en conduisant au cimetière, Ève dénonce ainsi ce qui a conduit son mari « tout droit $\underline{\text { au Cimetière » (p. 203): une incapacité à se contenter de ce que l'on a qu'elle considère à }}$ l'origine de toute ambition. Ève dénonce ainsi le manque de contentement [1'expression ne me semble pas très claire ici] dont les effets néfastes ont finalement conduit son mari « tout droit au Cimetière » (p. 203).Insatisfaction dont tout à la fois -elle s'exonère et inculpe sa fille

Moi, dit-elle, je sais qu'il faut savoir s'arrêter. Mais maintenant, pensai-je, elle ne peut plus s'arrêter, était-ce l'appel du cimetière où nous n'avons pas mis les pieds une seule fois en vingt ans, la voiture aussi roulait, elle n'avait pas besoin d'appuyer sur la pédale au contraire.

- Et finalement s'installer comme médecin et mourir. - Quels vilains souliers ! dit-elle.

Elle s'adressait soudain âprement à mes pieds (p. 198). 
Le saut abrupt de la mort du père aux pieds de la fille manifeste l'analogie que la mère aperçoit entre leurs désirs; la mère n'approuve ni l'endroit où sa fille «met les pieds » métaphoriquement (elle passe son temps à écrire) ni celui où elle les met littéralement (les souliers). Toutefois, non seulement l'avis de la mère selon lequel elle sait s'arrêter mieux que les autres membres de sa famille est contredit par ses propres actions, selon la narratrice, mais, de manière plus déconcertante, il l'est aussi par les actions de la voiture qu'elle conduit et qui cesse de répondre quand elle appuie sur la pédale : «Arrête ! dis-je. À ce moment-là ma mère dit : Eh ! Bien voilà que je ne peux plus m'arrêter. Et elle eut l'air effrayé. J'appuie sur le frein. Rien » (p. 199).

La concrétisation sous la forme d'une voiture capricieuse de l'idée abstraite selon laquelle la mère non plus ne commande pas le mouvement qu'elle a mis en marche rend impossible d'y voir une réflexion sur la psychologie spécifique d'Ève ; il est question plutôt du destin humain qui dicte que nos actions sont réglées par des volontés qui nous sont profondément inconnues. Or, ce destin auquel Ève n'échappe pas, est-il forcément « funèbre », comme l'avait évoqué la « faute » reconnue par la narratrice, ou peut-il promettre la vie autant que la mort? La suite insiste de plus en plus sur les contradictions qui marquent le déplacement en voiture des deux femmes. Le désir de ne pas avoir d'accident leur fait faire demi-tour, de sorte que le voyage vers le cimetière les conduit en fait dans la direction inverse ; la panne des freins cède inexplicablement la place à un défaut de l'accélérateur :

- Va plus vite, dis-je, la voiture monte comme une tortue. - Mais comment faire ? dit ma mère. Je ne trouve pas l'accélérateur, c'est tout ce qui manquait, j'appuie, j'appuie, pas de réponse. Nous n'avancions presque plus. Et sur l'autoroute. - Continuons, dis-je. - Pédicure ? Non, dit Tonpère. S'occuper des pieds ça n'était pas assez, dit ma mère. (p. 201)

Ce ne sont pas seulement la mère et la fille qui (se) continuent : l'écriture insiste sur la continuité entre le voyage et le récit, entre avancer et reculer, entre freiner et accélérer. Mais elle met en relief également la continuité entre métaphore et littéralité. En effet, la force de la séquence dépend en grande mesure de l'incertitude du statut de ce qu'elle raconte. La fin attire expressément l'attention sur son sens allégorique, en précisant que, par ce voyage qui s'était complètement retourné sur lui-même, contre lui-même, la narratrice et sa mère avaient «dessiné une des figures de [leur] destin : celle qui s'arrange pour tournoyer autour du cimetière inaugural » (p. 205). Cependant, le fait même d'expliciter le sens allégorique met en relief sa non-identité avec le sens littéral. Comme le souligne le côté fantastique du voyage 
que rien n'explique, ni donc ne normalise, l'effet métaphorique ne peut pas faire l'économie du sens littéral.

Ce voyage extraordinaire souligne ainsi que la métaphore est un voyage de la lettre : il n'y a pas d'effet métaphorique sans qu'une lettre se soit mise à la place d'une autre. La métaphore relève d'un «mouvement vital » dont Osnabrück suggère qu'il ne revitalise pas seulement la lettre déplacée. Certes, «tournoyer autour du cimetière inaugural », c'est reconnaître la centralité de la mort. Mais c'est aussi la tenir à distance. Paradoxalement, le destin peut aussi se redessiner, comme lorsque Pierre, le frère de la narratrice, décide subitement, à l'âge de cinquante-neuf ans, de ne plus être médecin :

Tu veux être dessinateur et voilà que la mère veut que tu sois dessinateur. Là-dessus tu deviens docteur et tu souffres toute ta vie de ne pas être qui tu aurais dû être. [...] Tu souffres toute ta vie d'une douleur qui commence par $d$ et finit par eur.

$[\ldots]$

- Et : J'ai écrit ma décision. [...] J'ai mis la lettre à la Bô̂te. [...] Il a suffi d'une lettre. Enfin moi j'étais le destinateur lui-même. (p. 208-209)

Afin de ne pas faire ce que sa mère voulait qu'il fasse, le frère avait passé sa vie à faire un métier que lui-même ne voulait pas faire. Or, tout aussi important que la substitution du $d$ oct-eur au $d$-essinat-eur sur laquelle attire l'attention la $d$-oul-eur «qui commence par $d$ et finit par eur», est le déplacement de «dessinateur» en «destinateur ». Il suffit en effet justement «d'une lettre » pour regagner une vie. Soulignons qu'écrire transforme Pierre non en auteur mais en «destinateur »; il ne s'agit pas de dessiner, fixer le destin (il ne sait pas ce qu'il va faire dorénavant), mais plutôt de le faire circuler.

La décision de Pierre le rapproche ainsi de sa sœur en regard de cela même qui la différencie de sa mère : «Elle ève, sans plus, tandis que moi je suis toujours tentée d'ajouter encore une lettre ou deux à sa syllabe» (p. 96). Pour nous tourner vers une conclusion, signalons un passage tardif où un tel ajout d'une lettre appelle la lecture en revenant sur certains des motifs qui nous ont déjà retenus. Il est question d'un des passages en italique où, au lieu de suivre littéralement la pensée «terre à terre » de sa mère, la narratrice prend la licence de lui prêter une voix plus poétique. La mère s'attriste de ne plus retrouver ses mots :

le mot MOT vient maintenant à la place de tout autre mot, dès que je veux dire l'un ou l'autre, la mort, car c'est Elle l'auteur de tous mes trous, me l'arrache de la chair à mots, preste, c'est chaque fois un geste d'arracheur de molaire, et à la place du disparu, s'affiche en bouchetrou le mot MOT. (p. 224) 
C'est du contraire même du mouvement «vital» métaphorique que souffre la mère, bien que la souffrance soit due au fait qu'un mot vient à la place d'un autre. Loin de faire travailler son sens littéral, le mot «mot » opère comme un passe-partout, un «bouchetrou » parfaitement substituable dont non seulement le corps propre n'entre pas en jeu, mais qui en plus vient faire violence au corps langagier, la «chair à mots ». Or, le contraste se remarque entre ce remplacement (thématique) qui chagrine Ève, et la parole imagée dans laquelle celleci s'en plaint. En plus de concrétiser l'absence du mot dans la figure d'une blessure physique, l'écriture l'accentue au niveau de la lettre, la « littéralise »: impossible de ne pas entendre le déplacement de «mot» à «molaire », en passant par «mort». «Molaire », c'est « mot » plus «l'r »; il suffit en effet d'une lettre pour que le « mot » donne une autre forme à la «mort »; pour que l'absence se renverse en supplément; pour que la mort soit déplacée, remplacée, dans et par la marche des mots.

Comment empêcher la mort d'être « l'auteur »? comment faire pour que quelque chose d'autre prenne sa place ? Voilà effectivement l'enjeu de cette citation. Si elle me semble propice pour clore mon propos, c'est que le prêt d'une voix poétique à la mère s'y oppose nettement au travail de la mort ; c'est surtout que la différence qu'elle met en jeu entre une parole qui néglige, voire écarte, la lettre, et une parole qui cherche au contraire à en exploiter l'irréductibilité, est manifestement au cœur des préoccupations de Cixous à travers Osnabrück dans son ensemble. Nous avons vu comment l'une des caractéristiques les plus marquantes d'Ève est son prosaïsme, auquel son mépris pour la «gloire» la fait tenir résolument. En essayant d'être fidèle à la mère sans que le portrait tombe dans l'imitation ou la répétition aride, Cixous s'est contrainte à tenter de rendre ce prosaïsme de manière à la fois prosaïque (littérale) et poétique (métaphorique). Cette tentative mène la narratrice à suivre Ève au plus près, transcrivant sa parole et l'accompagnant même quand elle partait dans la direction opposée à celle de ses propres inclinations. Mais elle se doit également de veiller à ce que son livre, qui comme tous les livres «pren[d sa] source au cimetière » (p. 140), reste vivant, « frais », au lieu de devenir la pierre tombale que cherche à ériger le discours maternel. Aussi cherche-t-elle, tout en emboîtant le pas à sa mère, à faire vivre les mots qu'elle lui consacre en les déplaçant, en en déplaçant le sens. Chez Cixous, prendre les mots au pied de la lettre, c'est en privilégier le mouvement, c'est carrément doter les lettres d'un pied; il convient de rappeler que la première scène racontée dans le prologue du livre finit justement par expliquer comment l'écriture donnait à la narratrice enfant sa place parmi «les jambes des lettres » (p. 12). Ce n'est sans doute pas un hasard si «l'r » qui distingue le «mot» [mo] de la «mort» $[\mathrm{moR}]$ est aussi ce qui fait la différence entre le «pied» [pje] et la «pierre » [pjer]. Il suffit 
« d'une lettre » pour que le sens change totalement, pour que l'immobilité même se mette en mouvement. Loin d'opposer littéralité et littérarité, Osnabrück, où l'on peut lire à la fois la littéralisation d'une métaphore et la métaphorisation de la lettre, représente une tentative aussi bien poétique que littérale de prendre les mots « au pied de la lettre ».

Mairéad HANRAHAN

University College London

m.hanrahan@ucl.ac.uk 